\title{
Effects of palonosetron on prolongation of corrected QT intervals may be less than reliable
}

Min et al. [1] investigated the effects of palonosetron, a second-generation 5-hydroxytryptamine-3 receptor antagonist, on corrected QT (QTc) intervals on electrocardiograms of patients undergoing laparotomies under sevoflurane anesthesia. Considering that prolongation of the QTc interval is associated with torsades de pointes, and palonosetron may at least theoretically increase QTc intervals by similar pharmacological mechanisms as ondansetron [2], clinicians will agree that an investigation into the effects of palonosetron on QTc intervals is required. Careful consideration is, however, needed, although Min et al. [1] concluded that palonosetron may induce QTc prolongation during general anesthesia with sevoflurane.

In the present study [1], there was a significantly different factor, famotidine, which also can influence the QTc interval. Consequently, it is difficult to exclude its effect on the QTc intervals, in contrast to the authors' description. Additionally, underlying diseases, electrolyte abnormalities, other drugs, adrenergic tone, temperature, and circadian variation, have other effects that may also result in prolongation of QTc intervals [3]. All of these influences may result in QTc prolongation, diminishing the effects of palonosetron alone. Furthermore, a less well-defined target population, risk of selection bias, and confounding factors in retrospective studies may affect the results [4]. Finally, Min et al. [1] concluded that palonosetron could induce QTc prolongation during general anesthesia with sevoflurane, whereas the prospective, randomized, and double-blinded study by Kim et al. [5] showed that preanesthetic administration of palonosetron $(0.075$ $\mathrm{mg}$ ) did not affect the QTc interval during the intraoperative period.

Thus, to better understand the effects of palonosetron on QTc intervals, it is essential to consider all of the factors related to QTc intervals, the weaknesses of retrospective studies, and related research results. Further well-designed studies on this issue from various perspectives will also be helpful.

\section{Jeounghyuk Lee and Junyong In}

Department of Anesthesiology and Pain Medicine, Dongguk University Ilsan Hospital, Goyang, Korea

E-mail: dragona1@dumc.or.kr

\section{References}

1. Min JJ, Yoo Y, Kim TK, Lee JM. Intravenous palonosetron increases the incidence of QTc prolongation during sevoflurane general anesthesia for laparotomy. Korean J Anesthesiol 2013; 65: 397-402.

2. Charbit B, Albaladejo P, Funck-Brentano C, Legrand M, Samain E, Marty J. Prolongation of QTc interval after postoperative nausea and vomiting treatment by droperidol or ondansetron. Anesthesiology 2005; 102: 1094-100.

3. Scuderi PE. Sevoflurane and QTc Prolongation: An Interesting Observation, or a Clinically Significant Finding? Anesthesiology 2010; 113: $772-5$.

4. Hess DR. Retrospective studies and chart reviews. Respir Care 2004; 49: 1171-4.

5. Kim HJ, Lee HC, Jung YS, Lee J, Min JJ, Hong DM, et al. Effect of palonosetron on the QTc interval in patients undergoing sevoflurane anaesthesia. Br J Anaesth 2014; 112: 460-8.

(C) This is an open-access article distributed under the terms of the Creative Commons Attribution Non-Commercial License (http:// creativecommons.org/licenses/by-nc/3.0/), which permits unrestricted non-commercial use, distribution, and reproduction in any medium, provided the original work is properly cited. 


\section{In Response}

We understand In's opinion. We performed the study to assess the effects of palonosetron on prolongation of the corrected QT intervals (QTc). We concluded that palonosetron might induce the prolongation of QTc. In our retrospective controlled study, we did not exclude patients who took other medicines, such as famotidine, which is known to affect the prolongation of QTc, because we measured the baseline QTc from the surgical record after monitoring during surgery in the operating theater. Although there was a significant difference between the two groups in terms of famotidine in our results, baseline QTc did not differ between the two groups. Moreover, the numbers of patients who were administered preoperative famotidine were higher in the control group than the QT prolongation group, even though famotidine can cause QT prolongation. Also, in fact, cases of QT prolongation induced due by famotidine are reported only rarely [1]. Our analysis could be subject to hidden biases, as we noted in the limitations section, since it was a retrospective study. We also hope that further well-designed studies on this subject will be performed.

Jeong Jin Min, Yongjae Yoo, Tae Kyong Kim, and Jung-Man Lee

Department of Anesthesiology and Pain Medicine, Seoul National University Hospital, Seoul National University College of Medicine, Seoul, Korea

\section{Jung-Man Lee}

Department of Anesthesiology and Pain Medicine, Seoul National University Hospital, Seoul National University College of Medicine, Seoul, Korea

E-mail: jungman007@gmail.com

\section{Reference}

1. Lee KW, Kayser SR, Hongo RH, Tseng ZH, Scheinman MM. Famotidine and long QT syndrome. Am J Cardiol 2004; 93: 1325-7. 